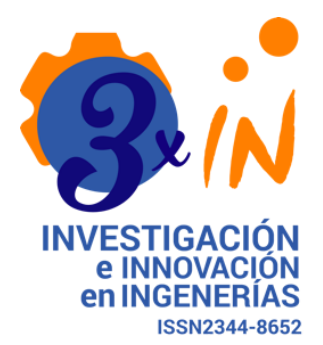

Open Access

Recibido:

12 de junio de 2018

Aceptado:

5 septiembre de 2018

Publicado:

11 diciembre de 2018

Correspondencia: mobustam@unal.edu.co, ajdazaa@unal.edu.co, pabustamanteba@unal.edu.co

DOI:

https://doi.org/10.17081/invinno.7.1 .2997

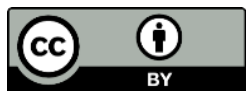

(c) Copyright: Investigación e Innovación en Ingenierías

\section{Determinación de la influencia del sodio superficial en la susceptibilidad de los mantos de carbón a combustión espontánea en una mina a cielo abierto}

\author{
Determination of the influence of surface sodium on the susceptibil- \\ ity of coal beds to spontaneous combustion in an open pit mine \\ Moisés Oswaldo Bustamante Rúa (iD) , Alan José Daza Aragón (iD, \\ Pablo Bustamante Baena (iD), Julián David Osorio Botero \\ Universidad Nacional de Colombia, Instituto de Minerales CIMEX, Colombia
}

Resumen

Objetivo: Identificar si la susceptibilidad a la combustión espontánea de carbón está relacionada por la influencia de la concentración de sodio superficial producto de la cercanía de los mantos de carbón a zonas costeras. Metodología: Se tomaron muestras de mantos de carbón de minería a cielo abierto de zonas ubicadas en diferentes lugares de Colombia, determinando su susceptibilidad a la combustión espontánea por sus registros históricos, luego se analizó la concentración de sodio en la superficie del carbón la cual fue obtenida utilizando una solución compuesta por $\mathrm{HCl}$ y $\mathrm{HNO}$ en concentraciones del $50 \%$, respectivamente, y se determinó la relación con la susceptibilidad y la cercanía costera. Resultados: Se encontró que no hay una relación directa entre la cantidad de sodio presente en superficie y la susceptibilidad del carbón, a su vez, no hay una correlación de los datos de concentración de sodio superficial y la distancia de los mantos a las costas lo que permitió demostrar que la teoría de compuestos iónicos no impacta en la combustión espontánea de carbón. Conclusiones: La variación de los datos de concentración de sodio superficial en la cantidad de mantos susceptibles y no susceptibles varía en un $15 \%$ mostrando que la susceptibilidad no tiene relación ni con la concentración de sodio en la superficie de las muestras ni con la cercanía costera

Palabras claves: Combustión espontánea, carbón, sodio superficial, pirofórico, minería.

\begin{abstract}
Objective: To identify if the susceptibility to spontaneous combustion changes is related to the influence of the concentration of surface sodium as a result of the proximity of coal beds to coastal areas. Methodology: Samples were taken from open-cast mining coal from areas located in different parts of Colombia, determining their susceptibility to spontaneous combustion by their historical records, then analyzing the concentration of sodium on the surface of the coal which was obtained using a solution composed of $\mathrm{HCl}$ and $\mathrm{HNO} 3$ in concentrations of $50 \%$, respectively, and the susceptibility and coastal proximity relationship was determined. Results: It was found that there is not a direct relationship between the amount of sodium, present in the surface, and the susceptibility of coal, in turn, there is no correlation of the data of surface sodium concentration and the distance of the mantles to the coasts which allowed to demonstrate that the theory of ionic compounds does not impact the spontaneous combustion of coal. Conclusions: The variation of the data of the surface sodium concentration on the amount of susceptible and non-susceptible beds correspondingly in a $15 \%$ that shows the susceptibility, has neither a relation to the concentration of sodium in the surface of the samples, nor to a proximity to the coast.
\end{abstract}

Keywords: Spontaneous combustion, coal, surface sodium, pyrophoric, mining.
Como citar (IEEE): M. Bustamante, A. Daza, P. Bustamante, J. Osorio, "Determinación de la influencia del sodio superficial en la susceptibilidad de los mantos de carbón a combustión espontánea en una mina a cielo abierto", Investigación e Innovación en Ingenierías, vol. 7, nº 1, 2019. DOI: https://doi.org/10.17081/invinno.7.1.2997 


\section{Introducción}

\section{Combustión espontánea de carbón}

El carbón es un material combustible de origen sedimentario, compuesto principalmente por una fracción orgánica denominada macerales [1, 2], y también por algunos minerales en mucha menor cantidad, que presentan contenidos de agua y gases en poros submicroscópicos. El carbón es producto de la acumulación y enterramiento de materia orgánica durante largos periodos de tiempo geológico. Estos restos orgánicos son afectados por una serie de procesos, que involucran la compactación del material enterrado inicialmente en cuencas de poca profundidad [2, 3, 4, 5]. Generalmente, los carbones se originan de la turba depositada en pantanos seguido del lignito; que después se trasforma a sub-bituminoso, para cambiar, a continuación, a bituminoso y, por último, a antracítico. El grado de transformación de la materia orgánica a través de la serie turba-lignitocarbones sub-bituminosos-bituminosos-antracita se desarrolla de forma natural y se conoce como carbonificación $[5,6]$. Esta se produce por la conjugación de múltiples variables, como la temperatura, ya que esta aumenta la cinética de las reacciones químicas de transformación del carbón $[7,8,9,10]$; además, la presión ayuda a reducir la porosidad y la humedad superficial mediante la compactación y el tiempo según el tipo de carbón producido. El grado del carbón mejora a medida que madura de turba a antracita.

El carbón es un material susceptible de combustión espontánea, ya que posee una temperatura crítica de autocalentamiento (Self-Heating Temperature), que es la temperatura mínima en la que los materiales se queman de forma espontánea [11, 12, 13, 14, 15], siendo, en consecuencia, el valor más bajo que se requiere para producir una sostenida reacción exotérmica. Por tal razón, si se llega a esta sin alcanzar el equilibrio térmico, la tasa de oxidación se acelerará [11,12].

La combustión espontánea de carbón es un fenómeno controlado principalmente por procesos de reacciones heterogéneas que se presentan en las ecuaciones:

$$
\begin{array}{ll}
2 \mathrm{C}+\mathrm{O}_{2} \rightarrow 2 \mathrm{CO} & \Delta \mathrm{H}^{0}{ }_{180^{\circ} \mathrm{C}}=-52,6 \mathrm{kcal} / \mathrm{mol} \\
\mathrm{C}+\mathrm{O}_{2} \rightarrow \mathrm{CO}_{2} & \Delta \mathrm{H}^{0}{ }_{180^{\circ} \mathrm{C}}=-94,1 \mathrm{kcal} / \mathrm{mol}
\end{array}
$$

La combustión espontánea es de gran preocupación en las minas de carbón del mundo, ya que provoca problemas ambientales como emisiones de gases contaminantes a la atmósfera, pérdidas económicas por el consumo de las reservas $[2,16]$, problemas de inestabilidad geotécnica del macizo rocoso [6] y problemas de salud pública por la cercanía con comunidades [17]. La susceptibilidad de la combustión espontánea del carbón en el 
mundo involucra algunos axiomas como la teoría de piritas, la teoría de compuestos iónicos salinos, la teoría de macerales, la teoría de adsorción de agua y la teoría de movimientos de tierra $[18,19,20,21]$.

Figura 1. Emisiones de gases a causa de la combustión espontánea de carbón

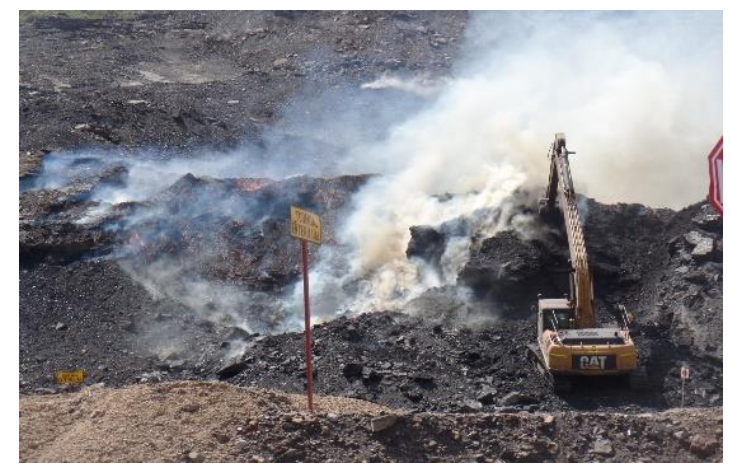

La combustión espontánea de carbón es un proceso de oxidación natural que se desarrolla gradualmente mientras ocurre un escalamiento en la temperatura [22,23]. El oxígeno es adsorbido por la superficie del carbón, generando una reacción química que resulta en la liberación de calor. Dicha tasa en la oxidación del carbón varía de 2 a 4 calorías por mol de oxígeno adsorbido [18]. En este sentido, por ser el sodio un elemento pirofórico puede contribuir al autocalentamiento del carbón si no se logran tasas efectivas de disipación de calor en el medio en el que se encuentra. El agua de mar contiene aproximadamente $11 \mathrm{ppm}$ de sodio, lo cual es el resultado de un proceso de millones de años, en el cual el sodio de las rocas se desprende a través de la erosión hasta llegar a los océanos. Este se presenta en forma de iones $\mathrm{Na}^{+}$cuando se encuentra en disolución [24]. Esta es la razón por la que en esta investigación se pretende analizar el impacto de la cercanía costera de las minas en el fenómeno de la combustión espontánea de carbón.

\section{Teoría de los compuestos iónicos}

Según esta teoría, elementos como el sodio, el litio y el potasio, los cuales hacen parte de los metales alcalinos y son conocidos como sustancias pirofóricas, reaccionan espontáneamente en presencia de oxígeno, agua o humedad del aire [21, 24, 25], desencadenando una reacción exotérmica violenta que facilita la combustión espontánea y la posible propagación en la totalidad del manto.

$$
\begin{aligned}
& 4 \mathrm{Na}+\mathrm{O}_{2} \rightarrow 2 \mathrm{Na}_{2} \mathrm{O} \quad \Delta \mathrm{H}^{0}{ }_{40^{\circ} \mathrm{C}}=-198,45 \mathrm{kcal} / \mathrm{mol} \\
& 2 \mathrm{Na}+2 \mathrm{H}_{2} \mathrm{O} \rightarrow 2 \mathrm{NaOH}+\mathrm{H}_{2} \quad \Delta \mathrm{H}^{0}{ }_{40^{\circ} \mathrm{C}}=-87,86 \mathrm{kcal} / \mathrm{mol}
\end{aligned}
$$

Las reacciones presentadas en las ecuaciones, donde se tiene la interacción del sodio con el oxígeno del aire y el vapor de agua, evidencian su exotermicidad, las cuales, comparadas con las que se tienen en la oxidación 
del carbón reflejadas en las ecuaciones son más altas. Esto contribuye en mayor medida en el autocalentamiento del carbón.

Ahora bien, las sustancias pirofóricas son sustancias, incluidas mezclas y soluciones (líquidas o sólidas), que, aun en pequeñas cantidades, se inflaman en pequeños intervalos de tiempo (segundos o minutos) tras entrar en contacto con el aire $y$, por tanto, son las que presentan la mayor tendencia a la combustión espontánea. El calentamiento espontáneo, por su parte, es un proceso en el que la reacción gradual de una sustancia con el oxígeno (del aire) genera calor [26]. Si la producción de calor es más rápida que la pérdida, la temperatura de la sustancia aumenta, y, después de un periodo de inducción, puede producirse la inflamación espontánea y la combustión [27]. Las reacciones químicas que generan calor de forma espontánea, al ser "fuentes internas de ignición", conllevan un riesgo de combustión, y el sodio es un material propenso al calentamiento espontáneo, el cual puede convertirse en fuente de ignición, provocando la combustión de materiales próximos como el carbón [28, 29].

\section{Termodinámica de la reacción de sodio}

A partir del diagrama de Van't Hoff que se presenta en la Figura 2, se observa que ambas reacciones de sodio son termodinámicamente factibles, debido a que la constante de equilibrio $(\mathrm{K})$ es alta y las presiones parciales de oxígeno y vapor de agua son cercanas a cero. El resultado de ello es que las reacciones tienden hacia la formación de óxido de sodio, hidróxido de sodio e hidrógeno gaseoso. Además, por ser positivas, las pendientes de las rectas indican que las reacciones son exotérmicas y, en consecuencia, generan una liberación de calor.

Figura 2. Relación de la constante de equilibrio con la temperatura de la propagación del carbón

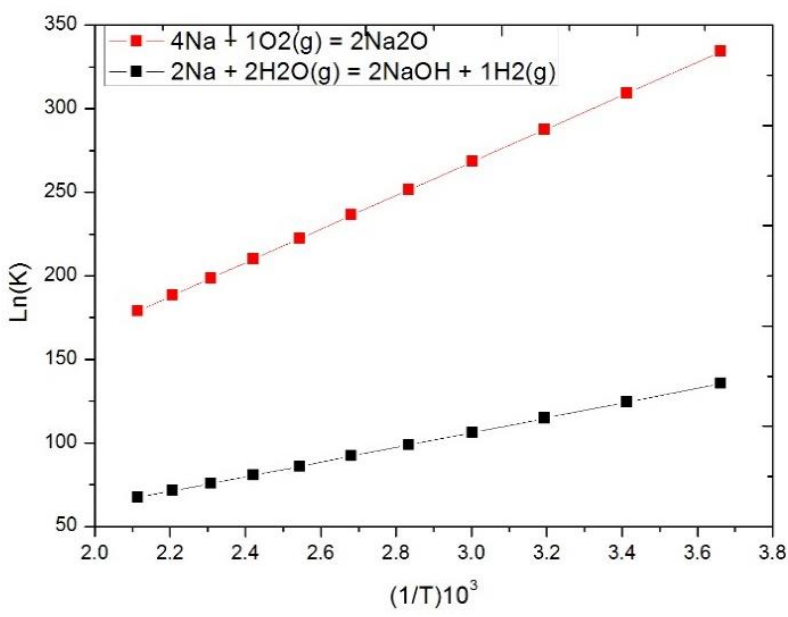




\section{Metodología}

Los puntos seleccionados para el análisis provienen de mantos de carbón de diferentes minas colombianas a cielo abierto, como se observa en la Figura 3. De estas, unas se seleccionaron por sus registros históricos referentes a la combustión espontánea de carbón y otras porque no la presentan. De acuerdo con esto, unas muestras provienen de mantos donde nunca se ha presentado el fenómeno de combustión espontánea, denominadas como Poco Susceptibles a Combustión Espontánea (PSCE); en tanto que las otras muestras provienen de mantos donde es muy frecuente la combustión espontánea de carbón, denominándose como Altamente Susceptibles a Combustión Espontánea (ASCE). De cada manto objeto de estudio se tomaron 15 muestras distribuidas sobre el área de influencia para conocer la variabilidad de los resultados obtenidos y la media muestral.

Figura 3. Zona de muestreo en una de las minas estudiadas

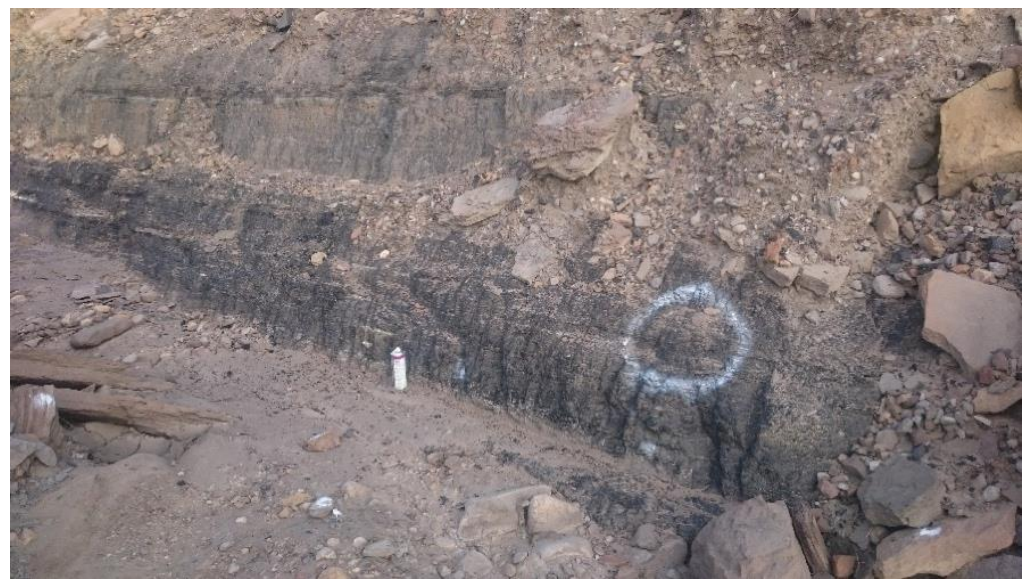

En la Figura 4, se presenta el análisis granulométrico de las muestras de carbón que se tuvieron en cuenta para el desarrollo del trabajo de investigación. Este resultado es producto de un proceso de conminución, en el cual se obtuvo un $\mathrm{d}_{25}$ de1 milímetro, un $\mathrm{d}_{50}$ de 1,75 milímetros, y un $\mathrm{d}_{80}$ de 2,36 milímetros, caracterizándose por ser una distribución monodispersa.

Figura 4. Análisis granulométrico de las muestras de carbón

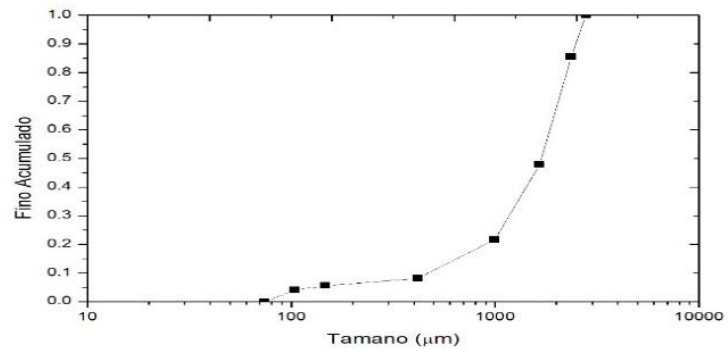

Los carbones seleccionados para el estudio corresponden al rango Bituminoso alto en volátiles $B$, según el sistema de clasificación de carbón ASTM, que poseen las características se presentadas en la Tabla 1. 
Determinación de la influencia del sodio superficial en la susceptibilidad de los mantos de carbón a combustión espontánea en una mina a cielo abierto

Tabla 1. Zona de muestreo en una de las minas estudiada

\begin{tabular}{|l|l|}
\hline \multicolumn{2}{|c|}{ Propiedades del carbón } \\
\hline Valor de Calentamiento (MJ/kg) & $26,9-28,5$ \\
\hline Humedad (\%w) & $8,3-10,3$ \\
\hline Volátiles (\%w) & $31,5-36,5$ \\
\hline Ceniza (\%w) & $5,5-11,5$ \\
\hline Sulfuros (\%w) & $0,4-0,8$ \\
\hline
\end{tabular}

\section{Procedimiento}

El procedimiento utilizado para el análisis de sodio en cada uno de los mantos objetos de estudio fue el siguiente:

1. Se realizaron 15 muestreos de canal en 14 mantos diferentes, conociendo previamente la susceptibilidad de cada uno a la combustión espontánea. Para cada muestra se tomaron aproximadamente 8 kilos, y se estableció una nomenclatura para su posterior identificación. Las muestras se obtuvieron mediante un corte uniforme y continuo perpendicular a la estratificación. Estas muestras de volumen de carbón triturado incluyen el espesor total del manto tomado, de tal manera que cada uno de los estratos constituyentes está representado en igual proporción a su espesor.

Figura 5. Zona de muestreo en una de las minas estudiadas

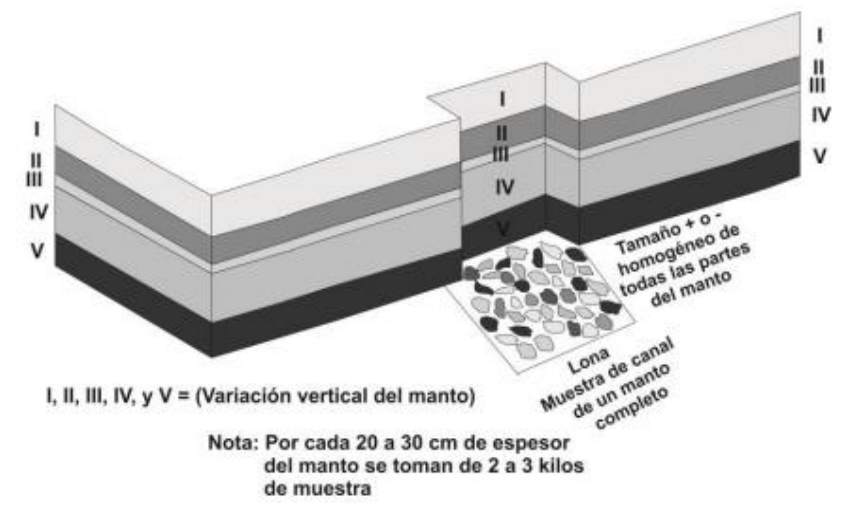

Fuente: [30]

2. Las muestras fueron llevadas al laboratorio para el respectivo análisis de sodio. Allí, inicialmente se realizó un cuarteo para cada una de las muestras seleccionadas con el fin de tomar, a su vez, una muestra representativa. Se emplearon dos ácidos diferentes: Ácido nítrico (HNO3) y ácido clorhídrico $(\mathrm{HCl})$, con el fin de cuantificar la cantidad de sodio que se encuentra adsorbido en la superficie del carbón [30].

3. Para cada prueba se utilizaron $50 \mathrm{~g}$ de carbón con un d80 de 2.36 milímetros, que se adicionaron a un beaker junto con los dos ácidos, 
cuyas concentraciones fueron de $50 \% \mathrm{HCl}$ y $50 \% \mathrm{HNO}$. La solución fue sometida a un agitador magnético de marca IKA RCT BASIC a 150 r.p.m. y se trabajó con una temperatura ambiente de $25^{\circ} \mathrm{C}$, la cual se mantuvo constante durante el periodo de prueba que duró cerca de 15 minutos.

Figura 6. Agitación de muestras de carbón para análisis de desorción de sodio

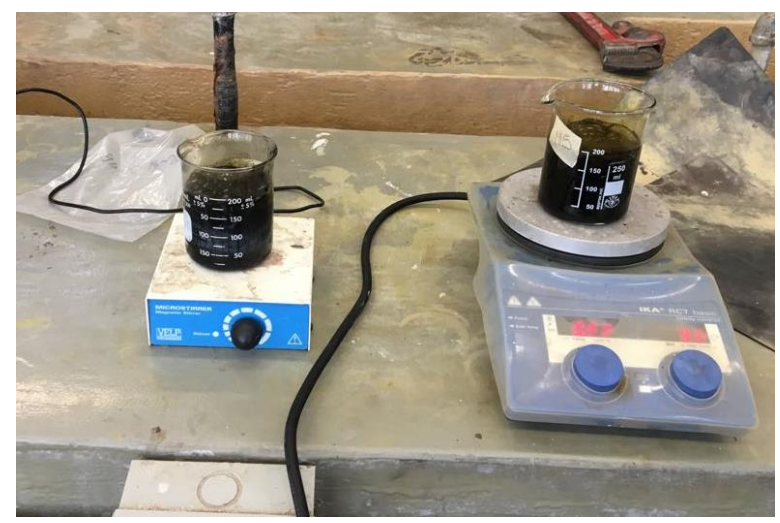

4. Cada prueba fue filtrada y analizada por medio de un ensayo de Espectrometría de Adsorción Atómica (AAS), realizado con el espectrómetro Thermo Scientific ICE 3000. Las series realizadas se presentan en las, que arrojaron la cantidad de sodio de la superficie del carbón en los diferentes mantos PSCE y ASCE.

Figura 7. Espectrómetro de adsorción atómica

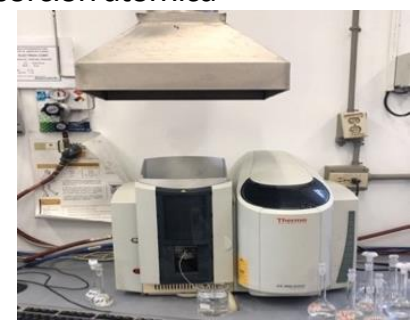

\section{Resultados y discusión}

En la Tabla 2 se presentan los resultados del análisis de sodio en la superficie del carbón para los diferentes mantos de estudio, en los cuales se presenta a su vez la condición de susceptibilidad a la combustión espontánea y cercanía a la costa. 
Determinación de la influencia del sodio superficial en la susceptibilidad de los mantos de carbón a combustión espontánea en una mina a cielo abierto

Tabla 2. Zona de muestreo en una de las minas estudiadas

\begin{tabular}{|c|c|c|c|}
\hline $\begin{array}{c}\text { Punto de } \\
\text { muestreo }\end{array}$ & Susceptibilidad & $\begin{array}{c}\text { Cercanía a la costa } \\
(\mathbf{k m})\end{array}$ & $\begin{array}{c}\text { Sodio en superficie } \\
\left(\mathbf{m g N a} / \mathbf{m}^{2}\right)\end{array}$ \\
\hline 1 & ASCE & 170 & 61,55 \\
\hline 2 & PSCE & 184 & 43,2 \\
\hline 3 & PSCE & 65 & 38,17 \\
\hline 4 & ASCE & 69 & 39,95 \\
\hline 5 & PSCE & 168 & 102,84 \\
\hline 6 & PSCE & 183 & 75,69 \\
\hline 7 & ASCE & 500 & 83,32 \\
\hline 8 & ASCE & 170 & 54,29 \\
\hline 9 & PSCE & 498 & 26,76 \\
\hline 10 & ASCE & 502 & 56,58 \\
\hline 11 & PSCE & 60 & 25,61 \\
\hline 12 & ASCE & 64 & 197,26 \\
\hline 13 & PSCE & 500 & 134,18 \\
\hline 14 & ASCE & 67 & 33,83 \\
\hline
\end{tabular}

Se utilizó el coeficiente de correlación para determinar la relación existente entre la concentración de sodio en la superficie del carbón y la cercanía del manto a la costa, como se observa, encontrándose muy poca correlación entre ambas variables. En concreto, se obtuvo un valor de 0,07 , lo que indica que la concentración de sodio no está relacionada con la cercanía del manto a la costa.

Figura 8. Diagrama de dispersión de la concentración de sodio y la cercanía a la costa

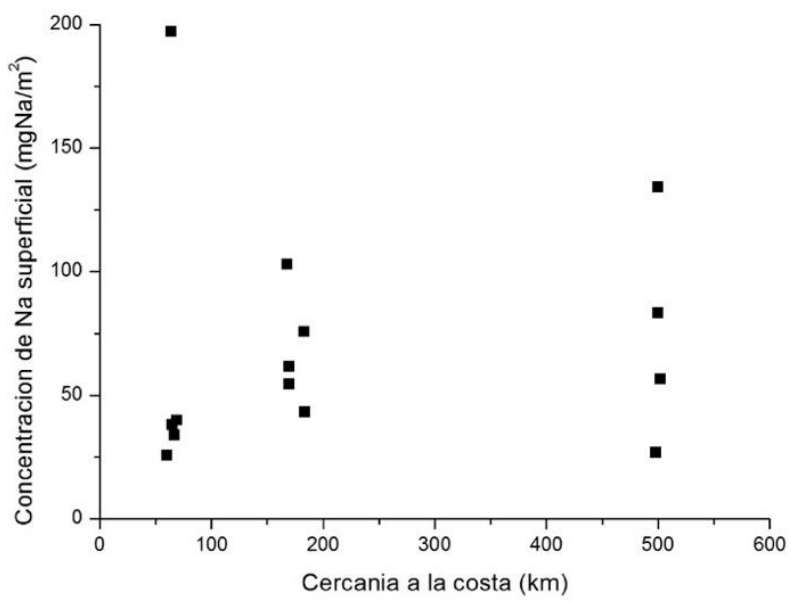

Por otro lado, la relación entre la concentración de sodio y la variable susceptibilidad se analizó a través de un diagrama de barras, encontrándose 
que la diferencia entre el valor acumulado de la concentración de sodio para mantos con alta susceptibilidad y poca susceptibilidad a la combustión espontánea es aproximadamente de15\%. Este porcentaje es poco representativo entre los dos tipos de mantos y no da pie para establecer que la concentración de sodio influye en la combustión espontánea de carbón.

Figura 9. Diagrama de barras entre la concentración de sodio acumulado y condición de susceptibilidad

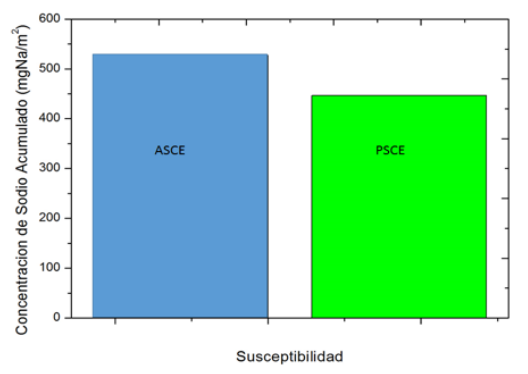

\section{Conclusiones}

Los resultados obtenidos del análisis por medio de espectrometría de adsorción atómica evidencian que las concentraciones de sodio en la superficie de las muestras susceptibles y no susceptibles pueden tener valores máximos de $197,26 \mathrm{mgNa} / \mathrm{m} 2$ y $134,18 \mathrm{mgNa} / \mathrm{m} 2$. También muestran que en los valores mínimos las concentraciones son de 33,83 $\mathrm{mgNa} / \mathrm{m} 2$ y $25,61 \mathrm{mg} \mathrm{Na} / \mathrm{m} 2$, respectivamente. Esto indica, en primera instancia, que no hay una relación directa entre la cantidad de sodio presente en superficie y la susceptibilidad del carbón.

Por su parte, los resultados de la gráfica de correlación de puntos muestra que la variable dependiente (causa) no se relaciona ni directa ni indirectamente con la variable independiente (efecto). Por este motivo, la concentración de sodio superficial presente en las muestras no se ve afectado por la distancia de los mantos de carbón a las costas, resultado que desmiente la hipótesis de que el sodio presente en las muestras es incrementado por la presencia de humedad o vientos marinos y que ésta concentración afecta la combustión espontánea de carbón.

Por último, la variación de los datos de concentración de sodio superficial en la cantidad de mantos susceptibles y no susceptibles varía en un $15 \%$, mostrando que la susceptibilidad no tiene relación con la concentración de sodio en la superficie de las muestras ni con la cercanía costera. El resultado es concluyente, y los datos levantados en la investigación muestran que la heterogeneidad de los mismos, cuando se analiza cualquier par de variables, se debe a la falta de correlación entre ellos, Esto significa que la concentración de sodio en las muestras, la susceptibilidad y la cercanía a las costas son variables independientes entre sí. 


\section{Referencias bibliográficas}

1. VJ. Cort, J. Vicente L. Cortés, Carbon. N Y, 1978.

2. M.O. Bustamante Rúa, A.J. Daza Aragón, P. Bustamante Baena, \& J.D. Osorio Botero, "Statistical analysis to establish an ignition scenario based on extrinsic and intrinsic variables of coal seams that affect spontaneous combustion", International Journal of Mining Science and Technology, 2018a DOI: //doi.org/10.1016/j.jjmst.2018.05.008

3. World Coal Institute. Coal, power for progress. 4th ed. London: 2016.

4. O.P. Gómez Rojas, I. Carmona Lopez. M.O. Bustamante Rúa, "Analysis of liberation of the groups of macerales of the coal:colombian coals", Bol Cencias La Tierra Cencias La Tierra, Vol. 21, $n^{\circ} .14,2007$.

5. Blandón Montes, Notas de clase de Caracterización avanzada de Carbones, 2015.

6. E. Díaz, J. Fernández, S. Ordóñez, N. Canto, A. González, "Carbon and ecological footprints as tools for evaluating the environmental impact of coal mine ventilation air", Ecol Indic, vol. 18, pp. 126-30, 2012. DOI:10.1016/j.ecolind.2011.11.009.

7. O. Levenspiel, Ingeniería de las reacciones químicas, Barcelona: Editorial Reverte S.A., 1986.

8. N.T. Moxon, S.B. Richardson, "Development of a self-heating index for coal", Journal Coal Preparation, vol. 2, n. 2, pp. 91-105, 1985.

9. M. Cortés Ibarra, R. Baribay "Temperatura", Acad. Bioinstrumental, 2015, p. 176.

10. J. Szekely, J. Evans, H.Y. Sohn, Gas-solid reactions, 1976.

11. J.D.N Pone, K.A.A. Hein, G.B. Stracher, H.J. Annegarn, R.B. Finkleman, D.R Blake, et al. "The spontaneous combustion of coal and its byproducts in the Witbank and Sasolburg coalfields of South Africa", Int J Coal Geol, vol. 72, pp. 124-40, 2007. DOI: 10.1016/j.coal.2007.01.001.

12. J.N. Carras, B.C Young, "Self-heating of coal and related materials: Models, application and test methods", Prog Energy Combust Sci, vol. 20, pp. 1 -15, 1994. DOI:10.1016/0360-1285(94)90004-3.

13. D.A Nield, A. Bejan. "Convection in porous media", Convect Porous Media, pp. 1-778, 2013. DOI: 10.1007/978-1-4614-5541-7. 
14. E. Kaymakçi, V. Didari. "Relations between coal properties and spontaneous combustion parameters", Turkish J Eng Environ Sci, vol. 26, pp. 59-64, 2002. DOI:10.1016/S0140-6701(03)90480-2.

15. G, Qi, D. Wang, K. Zheng, J. Xu, X. Qi, X. Zhong. "Kinetics characteristics of coal low-temperature oxidation in oxygendepleted air", J Loss Prev Process Ind, vol. 35, pp. 224-31, 2015. DOI:10.1016/j.jlp.2015.05.011.

16. J.A. Quintero, S.A. Candela, C.A Ríos, C. Montes, C. Uribe. "Spontaneous combustion of the Upper Paleocene Cerrejón Formation coal and generation of clinker in La Guajira Peninsula (Caribbean Region of Colombia)", Int J Coal Geol, vol. 80, pp. 196210, 2009. DOI:10.1016/j.coal.2009.09.004.

17. R.B. Finkelman, "Potential health impacts of burning coal beds and waste banks", Int J Coal Geol, vol. 59, pp. 19-24, 2004. DOI:10.1016/j.coal.2003.11.002.

18. P. Singh, An Investigation into Spontaneous Heating Characteristics of Coal and its Correlation with Intrinsic Properties An Investigation into Spontaneous Heating Characteristics of Coal and its Correlation with Intrinsic Properties, 2014.

19. H. Huo, X. Jiang, X. Song, Z. Li, Z. Ni, C. Gao, "Detection of Coal Fire Dynamics and Propagation Direction from Multi-Temporal Nighttime Landsat SWIR and TIR Data", Remote Sensing, vol. 6, n'. 2, pp. 12341259, 2014. DOI:10.3390/rs6021234.

20. Z. Liu, Y. Cheng, Q. Liu, J. Jiang, W. Li, K. Zhang, "Numerical assessment of $\mathrm{CMM}$ drainage in the remote unloaded coal body: Insights of geostress-relief gas migration and coal permeability", J Nat Gas Sci Eng, vol. 45, pp. 487-501, 2017. DOI:10.1016/j.jngse.2017.06.017.

21. W. Lu, Y.J Cao, JC. Tien. "Method for prevention and control of spontaneous combustion of coal seam and its application in mining field", Int J Min Sci Technol, vol. 27, pp. 839-46, 2017. DOI:10.1016/j.ijmst.2017.07.018.

22. Deng J, Zhao J, Zhang $Y$, Huang A, Liu X, Zhai X, et al. "Thermal analysis of spontaneous combustion behavior of partially oxidized coal", Process Saf Environ Prot, vol. 104, pp. 218-24, 2016. doi:10.1016/j.psep.2016.09.007.

23. Fierro V, Miranda JL, Romero C, Andres JM, Arriaga A, Schmal D, et al. "Prevention of spontaneous combustion in coal stockpiles 
Experimental results in coal storage yard", Fuel Processing Technology, vol. 59, Issue 1, pp. 23-34, 1999.

24. LENNETECH. Sodio (Na) y agua 2017:1. https://www.lenntech.es/sodio-y-agua.htm.

25. Smith KL, Smoot LD, Fletcher TH. Coal Characteristics, structure, and reaction rates. In: Fundamentals of coal combustion for clean and efficient use. Amsterdam: Elsevier Science Publishers.; 1993.

26. X. Qi, H. Xin, D. Wang, G. Qi. "A rapid method for determining the $R$ 70 self-heating rate of coal”, Thermochim Acta, vol. 571, pp. 21-7., 2013. DOI:10.1016/j.tca.2013.08.008.

27. Bestratén Belloví M, Piqué Ardanuy T. NTP 237: Reacciones químicas peligrosas con el agua. Instituto Nacional de seguridad e higiene en el trabajo, pp. 1-8., 2012.

28. Bustamante Rúa MO, Notas de clase de Introducción de sistemas heterogéneos 2016:40.

29. Su S, Chen H, Teakle $P$, Xue $S$. Characteristics of coal mine ventilation air flows. J Environ Manage 2008;86:44-62. doi:10.1016/j.jenvman.2006.11.025.

30. Universidad Nacional de Colombia. Instructivo para toma y manejo de muestras 2017:1-9. 